\title{
To the Question of the Natural-Scientific Basis of the Classification of Mental Diseases
}

\author{
Rosman SV* \\ Physician of Functional Diagnostics of SBIH, Regional Psychoneurological clinic, Russia
}

*Corresponding author: Rosman SV, Physician of functional diagnostics of SBIH, Regional psychoneurological clinic, Tver, Russian Federation, Russia.

Received Date: November 14, 2019

Published Date: November 19, 2019

\begin{abstract}
Based on new ideas about the implementation of information exchange in the brain, a new approach to objective diagnosis and natural-scientific systematics of mental diseases is proposed based on an innovative method of dispersion of amplitude-private characteristics of alpha-rhythm of EEG.

Keywords: Dispersion of amplitude-frequency characteristics of an alpha rhythm; Diagnosis of mental diseases; Paradigm shift of psychiatry

Abbreviations: DAFCAR: Dispersion of Amplitude-Frequency Characteristics of the alpha rhythm EEG; NGNB: Neuron-Glial Network of the brain; IDAFCAR: Indexes of Dispersion of Amplitude-Frequency Characteristics of the alpha rhythm EEG; CDAFCAR: Cartogram of Dispersion of Amplitude-

Frequency Characteristics of the alpha rhythm EEG
\end{abstract}

\section{Introduction}

We are entering an era of a new classification of mental illness - an ICD-11 that is unlikely to fundamentally solve all problems of psychiatry, as it will still be based on categorical ideas about the essence of psychopathology [1]. The question of reviewing these perceptions in psychiatry is a long-standing one, but he does not find his solution because there is no convincing evidence of correlation between objective neurophysiological research or pathological-anatomical changes in the brain and the clinic of psychopathology to date. The reason for this is the same fact - to consider all these problems from the old methodological positions almost the time of Hippocrates. According to these proposals, the disease must have an organic substrate whose changes are at the root of any disease: excess or deficiency of hormones, hypertrophy or hypotrophy of an organ or tissue, uncontrolled cell proliferation, etc. The neurophysiological process has a shorter history - since the XVII century but has not changed significantly since then information from the senses to the functional centers of the brain is transmitted like telegraph communication with signal switching in subcortical structures.

However mental diseases are not entered in these schemes. First, objective substrate is not found in them yet; secondly, secondly, normal linear information transfer is impossible as the brain is not capable to digital information processing owing to terrible functional inertia of nervous tissue and has no memory devices and databases. Attempts of representation of processing of information in the form of holograms do not maintain any criticism as these holograms need to be done of something and to store somewhere.

All these discrepancies indicate that concerning mental diseases there is no clear idea based on modern knowledge of an essence of information process.

It is unlikely this article can give irrefragable answer on so difficult questions - rather, this invitation to extensive discussion of a problem without which solution we will not be able to find the correct answers to those questions which it is necessary to us in such, without exaggeration, vital areas as psychology and psychiatry. It is possible even to speak about survival of the person and ways of its further Evolution. Our time - an era of information revolution which main maintenance is emergence of artificial intelligence. There is a set of the points of view on this process - from enthusiastic to apocalyptic. It is clear one - generally this problem is considered from purely outer side of opposition of 
Human Mind and Artificial Intelligence. The evolutionary nature of emergence of symbiotic social-technogenic information nature of this process is absolutely ignored [2].

And besides, the reason of it is misunderstanding of mechanisms of information exchange in a human brain. When their true character becomes clear to us, then we also will receive natural-science fundamentals of psychiatry. In the middle of the 19th century there was a concept according to which the psychopathology is a consequence of uniform process - "the theory of uniform psychosis" of Grizinger [3]. However the reason of it that the neurophysiology still is on positions of linear information transfer from receptors in a brain and commands about necessary actions from a cerebral cortex on the periphery was not succeeded to find out this process still, and. This "power vertical" which is dragged centuries from the social environment as the only possibility of management of executive structures, still interferes with the correct understanding of an essence of the happening information process in a brain.

A possible solution to the current impasse is the notion of a neurophysiological fractal self-collectible mechanism for internalizing afferent information - NFSMIAI (computer coding analogue). Despite the complex name, the essence of this mechanism is relatively simple. In the neural-glial network of the brain (NGNB) there are electrophysiologic processes caused by self-traumatic biochemical reactions of Belousov-Zhabotinsky, the external manifestation of which is the alpha rhythm of EEG. Normally, it has strict frequency characteristics from 9.5 to 12 $\mathrm{Hz}$ and certain phase shifts between individual areas of the brain. These oscillatory changes are the main (carriers) during interference with other oscillations that occur in the GM glial network as excitation passes through it. This process is iterativerecursive (by the re-entry mechanism), as a result of which fractal ordering of electrophysiological conditions around neurons occurs in the neurophysiological environment of the brain: some neurons end up in the zone of lowering the threshold of action potential, others in the zone of increase. It is in this way that neural associations predicted by P.K.Anokhyn. This is the internalization of afferent information (analogue of machine coding in PC). Action potentials from these neurons are transferred further, to centers of scenario formation in the way of human management. A behavior scenario is a set of neural associations in different control centers, corresponding to the nature of afferent information. Important here is the fact that the configuration of the electrophysiological fractal strictly corresponds to the nature of the afferent information, but in order for this condition to be met, the NGNB must maintain the stability of its properties. This stability is maintained by body homeostasis processes [4]. However, the complexity of this mechanism is its weak link - the increase of NGNB entropy due to natural and exogenous factors (invasion, intoxication, vascular pathology, injuries) causes NFSMIAI to begin to distort information.

This is the neurophysiological basis of psychopathology. The process of increasing the entropy of NFAMIAI can be easily monitored by studying the alpha rhythm parameters of EEG. For this purpose, a new method of investigation was created - dispersion of amplitude-frequency characteristics of alpha-rhythm (DAFCAR) [5]. Numerous studies carried out on this technique have justified the validity of Grizinger 's "theory of single psychosis": the basis of psychopathology is the disorganization of NGNB with the disruption of the internalization processes of afferent information and the disruption of the development of socially acceptable scenarios of personality behavior. The whole diversity of psychopathology can be easily explained by this process taking into account the degree of its expression, topical features and some nuances of violations in NGNB [6] (Figure 1).

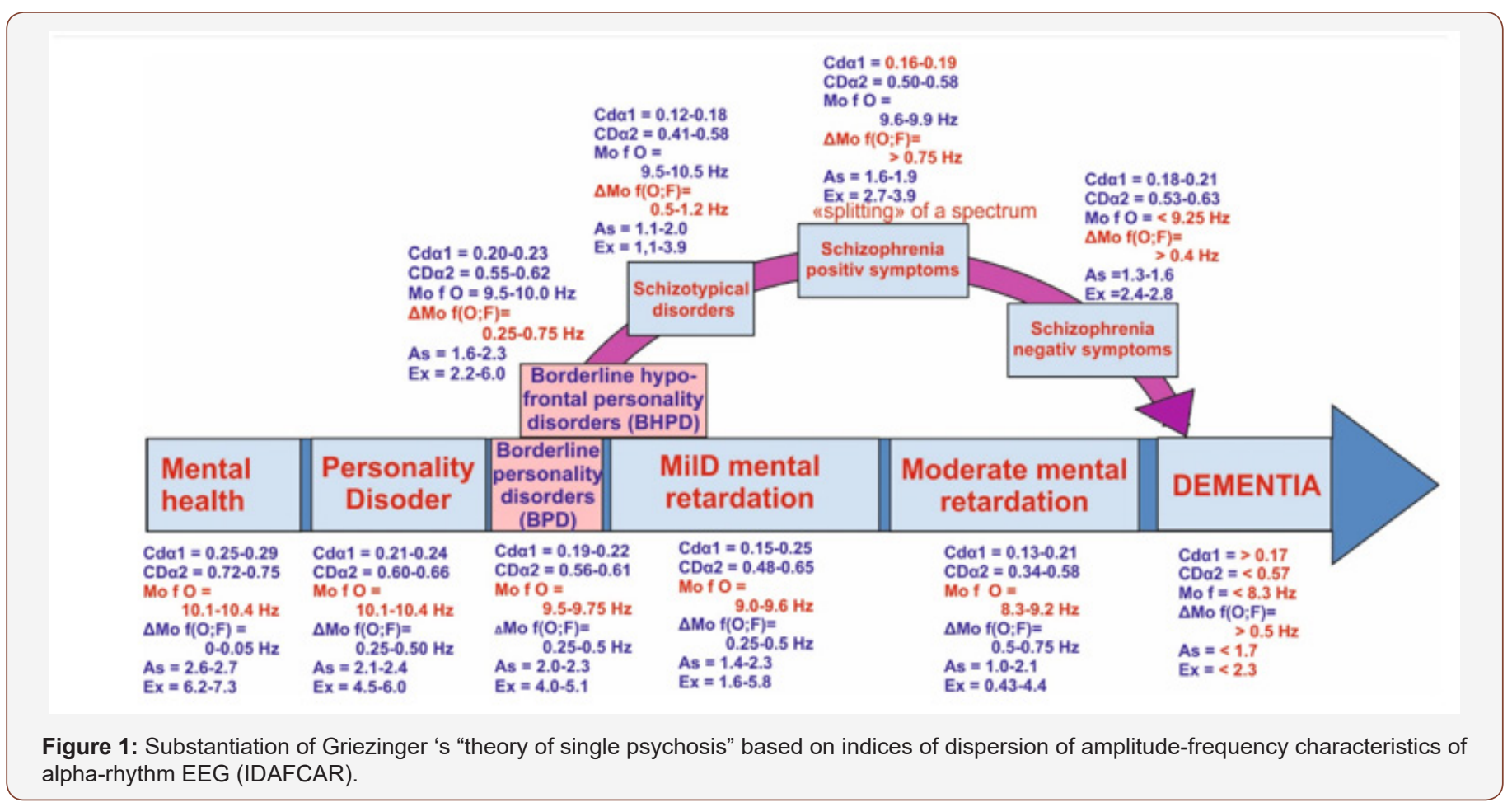


Another area of diagnosis of psychopathology based on IDACHAR is the imaging of these changes using DAFCAR - CDAFCAR dispersion cartograms. The following is an example of the differences of CDACHAR in normal and schizophrenia [7] (Figure 2).

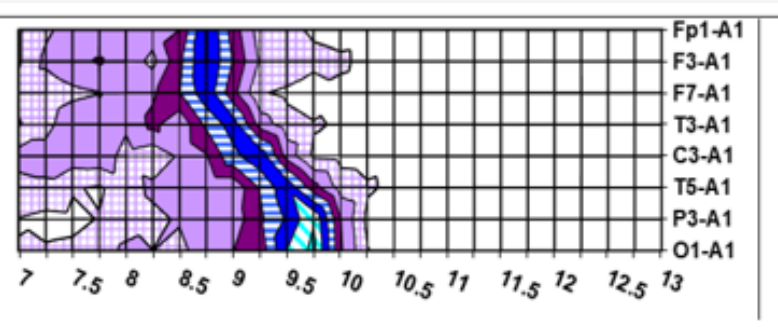

B

Figure 2: Comparative CDAFCAR is normal (A) and at schizophrenia (B). On a horizontal - the frequency of an alpha rhythm, $\mathrm{Hz}$; in vertical direction - assignments of EEG.

Actually, any psychopathology - process of increase of dementia, shown increase (or excessive acceleration) an alpha rhythm. Difference of diseases of a schizoid circle from usual dementia is regionality of these disturbances mainly in frontal lobes. In the final schizophrenia comes to an end with total delay of an alpha rhythm - defect.

\section{Naturally, there is a question: and how other nosological forms?}

It is all about improbable complexity of a configuration of potential damages of NGNB - it and defines all variety of clinical manifestations. Anyway, and it is necessary to treat damages to NGNB - not very well what localization. I already had to write that the depression is not independent process that damage of NGNB is its cornerstone. To treat a depression antidepressants - the same as to treat a carcinoma of the stomach drugs.

The question of obsessive-compulsive frustration, and also paroxysmal not epileptic mental diseases is represented very interesting. The proposed concept could provide an answer. As we indicated above, NGNB consists of two networks: neural and glial. Despite their functional unity, their specialization is somewhat different: the glial network creates a mechanism for perception of afferent information and its transformation into an electrophysiological spatial fractal (like a QR code), and the neural network creates neural associations of a conventional configuration with the probable possibility of "fixing" standard positions during training. In an iterative-recursive glial network, a «re-entry» mechanism is in effect, but the number of iterations is limited by special mechanisms (we can see them when a spikeslow wave type epileptiform activity occurs). In paroxysmal nonepileptic diseases, damage to the glial network causes disruption of this mechanism, and excitation begins to recycle nonstop, causing "automatic" neural associations that induce automatic actions. The same processes in the neural network, especially motor network, cause epilepsy.

Detailed consideration of all these problems can lead to the emergence of a natural-scientific basis of psychiatry and classification of mental diseases. But for this purpose it is necessary to develop a scientific base for studying the neurophysiological foundations of psychopathology using the DAFCAR method.

\section{DAFCAR technique scope}

1. Screening system of neurophysiological inspection within psychological-psychiatric examination of the complex contingent.

- The three-stage system of inspection is for this purpose offered:

A. The Neurophysiological Screening of Psychopathology (NSP) by means of the Detector of Neuromental Disorders device - takes about $5 \mathrm{~min}$. and does not demand for interpretation of highly skilled personnel [8]. Only persons with the revealed pathology need further inspection

B. Psychological testing - at the expense of preliminary NSP "elimination" of candidates can make up to $95 \%$

C. Consultation of the psychiatrist.

- The complex contingentare included:

a) children before enrollment of pupils

b) candidates for delivery of weapon, work in the specialties requiring special attention and responsibility

c) going to the universities

d) candidates for service in law enforcement agencies and recruits in VS

e) drivers, generally heavy-load and public transport

2. Diagnostics of mental diseases and control of the carriedout treatment, assessment of efficiency of pharmacological drugs; development of criteria of an extract of patients with mental diseases.

3. Forensic-psychiatric examination for the purpose of identification of boundary psychopathology, clinically not shown. 
4. Development of criteria for psychotherapeutic influences.

5. Scientific research for the purpose of studying of regularities of the mental processes at the personality correlating with neurophysiological changes.

\section{Tasks which need to be solved during further work}

1. Unification of EEG of researches with development of standard methods of shooting of EEG and the unified equipment

2. Development of the mathematical apparatus allowing to conduct polyposition correlation researches between IDAFCAR and clinic of psychopathology

3. Search of a possibility of automatic recognition of DCDAFCAR for creation of the database of images at different mental statuses for the purpose of more complete elimination of need of highly skilled personnel for interpretation of results of a research.

4. Search of the new patterns and IDAFCAR correlating with a mental status of the person.

5. Search of ways of coordination of neurophysiological problems and problems of creation of artificial intelligence within process of symbiotic process of society and artificial intelligence for the purpose of more successful use of information in improvement of human nature.

\section{Acknowledgement}

None.

\section{Conflict of Interest}

No conflict of interest.

\section{References}

1. Sanislow Charles A (2016) Updating the Research Domain Criteria. World Psychiatry 3(15): 222-223.

2. Yu S Zatuliveter (2005) The problems of control paradigm globalization in the mathematically uniform field of computer information. I. To the common function space. Probl Upr 2: 12-23.

3. Grisinger W (1861) Die Pathologie and Therapie der psychischen Krankheiten fiir Arzte und Studierende, Stuttgart, Germany.

4. Rosman S (2017) The Theoretical Foundations of Dispersion of Amplitude-Frequency Characteristics of the Alpha Rhythm of the EEG. Glob J Add \& Rehab Med 2(3): 555587.

5. Rosman S (2018) The «System 3 + 3» in a Problem of Searching of a New Paradigm in Psychiatry. Psychol Psychother Res Stud 1(5).

6. Rosman SV, Zhemov AG (2015) Modern view of the concept of "single psychosis". Mental health 5: 80-84.

7. Rosman S, Maximova N (2017) Paroxysmal-Progredient Paranoid Schizophrenia in the Context of Entropy Neuron-Glial Networks of the Brain. Glob J Add \& Rehab Med 4(1): 555628.

8. Maximova NE, Rosman SV, Spak LV, Zabodayev SV (2016) Possibilities of using alpha-rhythm dispersion for screening verification of mental diseases. Psychiatry 1: 16-25 